www.jmscr.igmpublication.org Impact Factor 5.244

Index Copernicus Value: 83.27

ISSN (e)-2347-176x ISSN (p) 2455-0450 crossref DOI: _https://dx.doi.org/10.18535/jmscr/v4i10.69

Journal Of Medical Science And Clinical Research

\title{
Emerging Issues Regarding Management of MDR Non-Fermenting Gram Negative Ventilator Associated Pneumonia in A Rural Catering Tertiary Care Hospital
}

\author{
Authors \\ Barnali Kakati, Sonika Agarwal, Shalini Gupta \\ Himalyan Institute of Medical Sciences, Dehradun, India \\ Corresponding Author \\ Sonika Agarwal \\ Deptt. of Critical Care Medicine, Himalyan Institute of Medical Sciences, SRHU \\ Swami Ram Nagar, Jolly Grant Dehradun 248016 \\ Email: Sonikaag1979@yahoo.com
}

\begin{abstract}
Introduction: Nosocomial infections due to Non-fermenting gram negative bacilli (NFGNB), especially multidrug resistant, are emerging as a great concern to the clinicians worldwide. NFGNB, normally a saprophyte cause serious infections in patients who are immune compromised or are hospitalized especially in ICU. The changing pattern of their susceptibility towards commonly used antibiotics specially carbapenems has necessitate to investigate the prevalent NFGNB circulating in a particular setting and their susceptibility in order to implicate appropriate empirical treatment. In this study we determined the antibiotic sensitivity pattern of the NFGNBs isolated from respiratory secretions of the patients in our ICU.
\end{abstract}

Design: Observatinal cross-sectional study.

Setting: Intensive care unit of a rural catering tertiary care hospital.

Material and Methods: A total of 523 respiratory tract samples were obtained from patients admitted to ICU during a period of 6 months from September 2015 to March 2016.The samples were processed as per CLSI guidelines on blood agar and macconkey agar. Bacterial growth was identified up to species level and antimicrobial susceptibility testing was determined by Vitek 2 compact (BioMérieux, France).

Result: Out of 150 patients with Ventilator associated pneumonia due to non-fermenting gram negative bacilli 63\% were male and maximum age effected is 45-75 yrs with medical disease and multiple comorbidities. Acinetobacter baumanii was most common isolate (54.66 \%) followed by Pseudomonas aeruginosa(28.66\%), Elizabethkingia meningoseptica(8.66\%) and Strenotrophomonas maltophilia(8\%). Antibiotic resistance among non-fermenting gram negative bacilli appears to be high.

Conclusion: Surveillance of nonfermenting gram negative bacilli infections in ICU appears to be important as organism causing infection and their sensitivity profile changes from hospital to hospital and over the period of time.

Keywords: $N F G N B, I C U, V A P, V I T E K 2$. 


\section{Introduction}

Ventillator associated pneumonia (VAP) is one of the most common and most challenging infections in ICU as they are difficult to treat and increases duration of stay in ICU leading to increased mortality in ICU ranging from $20 \%$ to $70 \% .{ }^{[1]}$, ${ }^{[2],[3]}$ Among the causative agents of VAP, isolation of non fermenting gram negative bacilli are becoming a cause concern to the clinicians due to their multidrug resistant property. Nonfermenting gram negative bacilli are aerobic nonspore forming saprophytes which do not utilize glucose as a source of energy or utilize it after oxidation and gives positive cytochrome oxidase test. ${ }^{[4]}$ They are ubiquitous in nature and can be found in soil and water and can survive on inanimate structures for long time in a energy deficit environment. They can be spread horizontally through fomites or through hands of health care workers. They are usually resistant bugs which develop resistance through various mechanisms like enzyme production, over expression of efflux pump, porin deficiency or target site alteration. ${ }^{[5]}$ Most common nonfermenting gram negative organisms are Pseudomonas and Acinetobacter but now other types are also increasing and their sensitivity pattern are also changing. So, there is a need to monitor and document the occurrence and drug resistant pattern of NFGNB in our ICU which in turn can help us to advocate empirical therapy in these cases. With this background this study was conducted to see the recent trends of nonfermenting gram negative infections causing VAP and their sensitivity pattern.

\section{Materials and Methods}

After taking approval from research and ethical committee of our institute this was a cross sectional study conducted in ICU of multi specialty hospital over a period of 6 months between September 2015 to march 2016. During the astudy period total of 523 respiratory tract samples were received from ICU. A total number of 386 ET secretions, 110 TT secretions and 27 BAL specimens were received in the microbio- logy laboratory from patients of different age groups and sex admitted in ICU suspected of suffering from lower respiratory tract infections. The specimens were processed for bacterial pathogens, in a semi quantitative manner.

\section{Sample processing}

All the samples were processed as per standard technique and after confirming the sample to be representative of lower respiratory tract secretion samples were cultured on blood agar and MacConkey agar plates. Single or mixed growth (two or more than two isolates per specimen) isolated from all the consecutive samples were identified up to species level and their antimicrobial susceptibilities were determined by using Vitek 2 compact (Bio Mérieux, France). American Type Culture Collection (ATCC) strains were used as control strains. The result of susceptibility testing was interpreted as per CLSI guidelines. $^{[6]}$

\section{Result}

Out of 523 samples 150 were found to be positive for non- fermenting (NF) gram negative organisms from respiratory secretion samples. Male: Female ratio among the patients having infection with NFs was found to be 1.6:1. With age there seems to be increasing trend of infections with NFGN bacilli maximum in 45-60 yrs age group especially in medical patients with co-morbid illness as depicted in table 1 and 2.

Table 1: Age and sex distribution of patients

\begin{tabular}{|l|l|}
\hline Sex \\
\hline Male & $94(62.6 \%)$ \\
\hline Female & $56(37.33 \%)$ \\
\hline Age & $0(0 \%)$ \\
\hline $0-15(\mathrm{yrs})$ & $25(16.66 \%)$ \\
\hline $15-30(\mathrm{yrs})$ & $30(20 \%)$ \\
\hline $30-45(\mathrm{yrs})$ & $43(28.66 \%)$ \\
\hline $45-60(\mathrm{yrs})$ & $42(28 \%)$ \\
\hline $60-75(\mathrm{yrs})$ & $10(6.66 \%)$ \\
\hline$>75(\mathrm{yrs})$ &
\end{tabular}

Table 2: Diagnosis wise distribution of patients

\begin{tabular}{|l|l|}
\hline Medical & $112(74.6 \%)$ \\
\hline Surgical & $48(32 \%)$ \\
\hline
\end{tabular}

Distribution pattern of NFs is depicted in table 3 . Acinetobcter Baumanii was most common isola- 


\section{JMSCR Vol||04||Issue||10||Page 13232-13238||October}

ted non- fermenting bacteria i.e 82(55\%) followed by Pseudomonas aeruginosa 43 (28.66\%), Elizabethkingia meningioseptica 13 (8.66\%) and Strenotrophomonas maltophilia 12(8\%).

Table 3: Distribution of organism in the samples

\begin{tabular}{|l|l|}
\hline \multicolumn{2}{|l|}{ PERCENTAGE OF ORGANISM ISOLATED } \\
\hline A.baumanii & $82(54.66 \%)$ \\
\hline P.aeruginosa & $43(28.66 \%)$ \\
\hline E.meningoseptica & $13(8.66 \%)$ \\
\hline S.maltophilia & $12(8 \%)$ \\
\hline
\end{tabular}

Antibiotic sensitivity pattern as depicted in table 4. A. baumanii had shown maximum sensitivity to colistin/polymyxin B followed by tigecycline while most of them were found to be resistant to carbapenems. P.aeruginosa was most sensitive to colistin/polymyxin B with moderate sensitivity to cefepime, aztreonam, carbapenems, levofloxacin and amikacin. E.meningoseptica had shown good sensitivity to cotrimaxazole, clindamycin and vancomycin. S.maltophilia had shown $100 \%$ sensitivity to levofloxacin and cotrimaxazole. E.Meningoseptica and S.maltophilia are intrinsically resistant to carbapenems so sensitivity pattern is not applicable.

Table 4: Antibiotic sensitivity profile of organism isolated

\begin{tabular}{|c|c|c|c|c|c|c|c|c|c|c|c|c|}
\hline Antibiotics & A.Baumanii & & & P.aerugino & & & E.menir & osept & & S. malto & ilia & \\
\hline & $\mathrm{S}$ & I & $\mathrm{R}$ & $\mathrm{S}$ & I & $\mathrm{R}$ & $\mathrm{S}$ & I & $\mathrm{R}$ & $\mathrm{S}$ & I & $\mathrm{R}$ \\
\hline Ampi-sul & $\begin{array}{l}12 \\
(14.63 \%)\end{array}$ & 0 & $65(79.2 \%)$ & 0 & 0 & $10(23.25 \%$ & 0 & 0 & 0 & 0 & 0 & 0 \\
\hline Ticarcillin & 0 & 0 & $\begin{array}{l}25(30.48 \\
\%)\end{array}$ & 0 & $\begin{array}{l}15(34 . \\
8 \%)\end{array}$ & $10(23.25 \%$ & 0 & 0 & 0 & $\begin{array}{l}10(83.8 \\
3 \%)\end{array}$ & 0 & 0 \\
\hline Piperacillin & 0 & 0 & $25(30.4 \%)$ & $24(55 \%)$ & 0 & $15(34.8 \%)$ & $\begin{array}{l}2(15.3 \\
8 \%)) \\
\end{array}$ & 0 & 0 & 0 & 0 & 0 \\
\hline Pip-tazo & $\begin{array}{l}25(30.48 \\
\%)\end{array}$ & 0 & $\begin{array}{l}60(73.14 \\
\%)\end{array}$ & $\begin{array}{l}28(65.11 \\
\%)\end{array}$ & 0 & $18(41.8 \%)$ & 0 & 0 & 0 & 0 & 0 & 0 \\
\hline Cefoxitin & 0 & 0 & $\begin{array}{l}25(30.48 \\
\%)\end{array}$ & 0 & 0 & $10(23.3 \%)$ & 0 & 0 & 0 & 0 & 0 & 0 \\
\hline Ceftazidime & $4(4.87 \%)$ & $\begin{array}{l}3(3.65 \% \\
)\end{array}$ & $62(75.6 \%)$ & $\begin{array}{l}16(37.20 \\
\%)\end{array}$ & 0 & $33(76.6 \%)$ & 0 & 0 & $7(53.84 \%)$ & 0 & 0 & 0 \\
\hline Cefo-sul & $\begin{array}{l}10 \\
(12.19 \%)\end{array}$ & $8(9.7 \%)$ & $64(78 \%)$ & $\begin{array}{l}11(25.5 \% \\
\end{array}$ & 0 & $15(34.9 \%)$ & $\begin{array}{l}2(15.4 \\
\%)\end{array}$ & 0 & 0 & $3(25 \%)$ & 0 & $8(66.6 \%)$ \\
\hline Cefepime & $5(6.02 \%)$ & 0 & $73(89 \%)$ & $\begin{array}{l}16(37.2 \% \\
\end{array}$ & 0 & $20(46.5 \%)$ & 0 & 0 & $3(23 \%)$ & 0 & 0 & $10(83.33 \%)$ \\
\hline Aztreonam & 0 & 0 & $50(70 \%)$ & $28(65 \%)$ & 0 & $15(35 \%)$ & 0 & 0 & 0 & 0 & 0 & 0 \\
\hline Ceftriaxone & 0 & 0 & $7692.7 \%()$ & 0 & 0 & $20(46.5 \%)$ & 0 & 0 & 0 & 0 & 0 & 0 \\
\hline Imipenem & $4(4.87 \%)$ & $4(4.8 \%)$ & $74(90.2 \%)$ & $\begin{array}{l}20(46.5 \% \\
\end{array}$ & 0 & $23(53.4 \%)$ & NA & NA & NA & NA & NA & NA \\
\hline Meropenem & $6(4.3 \%)$ & $1(1.2 \%)$ & $76(92.6 \%)$ & $\begin{array}{l}22(51.16 \\
\%)\end{array}$ & 0 & $21(48.8 \%)$ & NA & NA & NA & NA & NA & NA \\
\hline Amikacin & 0 & 0 & $70(85 \%)$ & $\begin{array}{l}18(41.86 \\
\%)\end{array}$ & 0 & $25(59 \%)$ & 0 & 0 & $13(100 \%)$ & 0 & 0 & $9(75 \%)$ \\
\hline Levofloxacin & $2(2.4 \%)$ & $\begin{array}{l}4(4.87 \% \\
)\end{array}$ & $76(92.6 \%)$ & $17(39.5 \%$ & $\begin{array}{l}5(11.6 \\
\%)\end{array}$ & $21(48.83 \%$ & $\begin{array}{l}2(15.4 \\
\%)\end{array}$ & 0 & $11(84.6 \%)$ & $\begin{array}{l}12(100 \\
\%)\end{array}$ & 0 & 0 \\
\hline Tigecycline & $60(73 \%)$ & $\begin{array}{l}10(12.2 \\
\%)\end{array}$ & $12(14.4 \%)$ & NA & NA & NA & 0 & 0 & 0 & 0 & 0 & 0 \\
\hline $\begin{array}{l}\text { Colistin/poly } \\
\text { B }\end{array}$ & $82(100 \%)$ & 0 & 0 & $43(100 \%)$ & 0 & 0 & 0 & 0 & 0 & NA & NA & NA \\
\hline Cotrimaxazole & $\begin{array}{l}10(12.19 \\
\%)\end{array}$ & 0 & $72(87 \%)$ & 0 & 0 & $43(100 \%)$ & $\begin{array}{l}11(84 . \\
6 \%)\end{array}$ & 0 & 0 & $\begin{array}{l}12(100 \\
\%)\end{array}$ & 0 & 0 \\
\hline $\begin{array}{l}\text { chloremphenic } \\
\text { ol }\end{array}$ & $11(13.4 \%)$ & 0 & $71(86.5 \%)$ & $4(9.3 \%)$ & 0 & $25(58 \%)$ & 0 & 0 & 0 & 0 & 0 & 0 \\
\hline Clindamycin & NA & NA & NA & NA & NA & NA & $\begin{array}{l}12(92 . \\
3 \%)\end{array}$ & 0 & 0 & NA & NA & NA \\
\hline Vancomycin & NA & NA & NA & NA & NA & NA & $\begin{array}{l}13(100 \\
\%)\end{array}$ & 0 & 0 & NA & NA & NA \\
\hline
\end{tabular}

\section{Discussion}

In the critically ill patients gram-negative pathogens, with the greatest burden are the non-fermenting bacteria Acinetobacter baumannii and P. aeruginosa, for which few therapeutic options are available. Non-fermenting Gram-negative bacilli are ubiquitous in the envir- onment. Pseudomonas aeruginosa is the most frequently isolated micro-organism, followed by Acinetobacter baumannii and Stenotrophomonas maltophilia. Non-fermenters may differ in their virulence and transmissibility, but most of them have potential to develop and transfer multidrug 
resistance. The resistance pattern of nasocomial bacilli varies from country to country worldwide and within the same country over the period of time ${ }^{[3]}$. For this reason, accurate identification of non-fermenters to species level and their sensitivity pattern is of great value for appropriate patient management.. Non-fermenters can cause a vast variety of infections. The main clinical syndromes due to non fermenting gram negative infections esp acinetobacter in ICU patients include pneumonia and bacteremia. ${ }^{[2]}$

Nasocomial infections are more common in elderly age group as they have low immunity and in medical patients with multiple comorbidities. The NFGN baciili are although considered as commensals with low virulence but can cause difficult to treat life threatening infections specially in state of immune supression, cystic fibrosis, indwelling catheters, invasive diagnostics and therapeutic procedures, prolong hospital stay and indiscriminate use of multiple antibiotics. ${ }^{[7],[8]}$ Their pathogenic potential has been well established by frequent isolation and their relation with clinical disease specially in ICU settings causing VAP. In our study lower respiratory tract infection caused by NFGN bacilli in ICU setting was more common in age group 45-60 yrs and more common in male which was similar in study by Ridhima et al and Sarika et al. ${ }^{[9,10]}$

Acinetobacter baumannii is a gram negative cocobacilli with oppurtunistic pathogenic potential which can acquire resistance by various mechanisms in response to challenges with new antibiotics. Despite having low virulence MDR acinetobacter have an extremely high crude mortality rate and occurs most frequently in critically ill patients. It poses a formidable threat to ICU patients. ${ }^{[13]}$. A. baumanii is gaining more attention now a days due to its ability to form biofilm, survival properties, increased virulence and antibiotic resistance In our study $A$. baumanii was the most frequent isolate from respiratory tract sample around 55\% cases which was similar to study by ridhima et al from delhi[9] who also studied respiratory samples. A. baumanii which were isolated were mostly pan drug resistant sensitive only to colistin and tigecycline. Tigecycline resistantce was seen in 12 isolates about $14.4 \%$ cases which is of concern but the most worrisom was carbapenem resistance which was seen in $>90 \%$ cases. Ana milda castern et al showed that resistance of acinetobacter to carbapenem is on rise and was found to be as high as $77 \%$ to imipenem and $80 \%$ to meropenem ${ }^{[12]}$ Carbapenem resistance depends upon the length of stay in ICU and selection pressure due to misuse of carbapenems. Colistin seems to be the only effective and baseline antibiotic due to increase in carbapenem resistance in our setting which is similar to Oliviera MS et al ${ }^{[14]}$ Colistin use has its disadvantage being nephrotoxic and neurotoxin and development of colistin resistance is a serious concern ${ }^{[14]}$

$P$. aeruginosa is a ubiquitous organism with ability of to survive on minimal nutritional requirements and to tolerate a variety of physical conditions has allowed this organism to persist in both community and hospital settings. $P$. aeruginosa is especially problematic for seriously ill patients in ICUs as it increases morbidity, mortality, length of stay and hospital expenses .The antibiotic susceptibility patterns of $\mathrm{P}$. Aurogenosa may change with time and may vary from hospital to hospital. Susceptibility patterns changes due to resistance transfer and mutant selection from misuse of antibiotics ${ }^{[15]}$. In our study $P$. aeruginosa was moderately sensitive around $50 \%$ to levofloxacin, amikacin, carbapenems, aztreonam, pip-tazo, ceftazidime and cefoperazone sulbactum and $100 \%$ sensitive to colistin. which is in concordance with study by kalidas et al ${ }^{[11]}$ and Deepak juyal et al ${ }^{[12]}$

S. maltophilia is a Gram-negative obligate aerobe which is rod shaped and motile with a few polar flagella. It has the ability to live in nutrient-poor aqueous environments for long time. Stenotrophomonas maltophilia is an important nosocomial pathogen associated with infections of compromised individuals. Generally associated with infections of the respiratory tract, it also has 
potential to cause bacteremia, endocarditis , and urinary tract infections. S. maltophilia is intrinsiccally resistant to multiple antibiotics including carbapenems and disinfectants. The drug resistance mechanisms are acquired by the horizontal transfer of antibiotic resistance through plasmids, transposons, integrons, integron-like elements, insertion element common region (ISCR) elements, and biofilms [16] Multidrug resistant (MDR) strains are also readily selected from susceptible $S$. maltophilia in the laboratory . That is why a major predisposing factor for $S$. maltophilia infection is prior antibiotic usage ${ }^{[17]}$. In our study S.maltophilia was isolated in $8 \%$ of cases which was similar to data from the SENTRY Antimicrobial Surveillance Program during 1997 to 2008 revealed a $3.1 \%$ rate of recovery of S.maltophilia from hospitalized patients with pneumonia from 2004 to 2008 , with regional recovery rates of $3.3 \%$ for the United States, $3.2 \%$ for Europe, and $2.3 \%$ for Latin America ${ }^{[17]}$. In our study S. Multophilia was found to be $100 \%$ sensitivity to levofloxacin and trimethoprim sulphamethoxazone and good sensitivity to ticarcillin and tigecycline while $33.33 \%$ were sensitive to ampicillin sulbactum, piperacillin tazobactum,cefoperazone sulbactum. So cotrimaxazole appears to be baseline therapy for treatment of S.maltophilia casing VAP.

Elizabethkingia meningoseptica is a nosocomialadapted Gram-negative bacillus intrinsically resistant to common antibiotics used in the intensive care setting. ${ }^{[19]}$ Patients at high risk of E. meningoseptica infections include preterm children, the immunosuppressed and patients exposed to antibiotics in critical care units and on dialysis $^{[18]}$. Strict infection control policies are required to control infections as saline, lipid, and chlorhexidine gluconate solutions as well as contaminated sinks have been implicated as sources of infection following outbreak investigations ${ }^{[20]}$. In our study $85 \%$ were sensitive to cotrimaxazole while more than $92 \%$ sensitivity with clindamycin and $100 \%$ sensitivity to vancomycin was observed. Resisitance to levofloxacin, piperacillin tazobactum was high. Vancomycin was once considered as drug of choice but studies have revieled high MIC for vancomycin for E.meningoseptica meningitis. E.meningoseptica is intrinsically resistant to colistin and carbapenems. So non-judicious use of carbapenems and colistin act as selection pressure for the growth of these organisms. so to control outbreaks of E.meningoseptica infections in ICU strict infection control strategies with judicious use of antibiotics and MIC based treatment is required.

A.baumanii and P.aeruginosa appears to be major and emerging cause of VAP in ICU setting in our study. Antibiotic resistance is common with these infections. Carbapenem backbone appears to be broken in management of these infections and colistin appears to be baseline and most effective antibiotic in these infections either alone or in combination. As the causative organisms and their susceptibility profile changes from time to time and from hospital to hospital so continous survillence of the data is required. Adequate infection control policies is required to control infections caused by NFGN bacilli and judicious use of antibiotics using $\mathrm{Pk} P \mathrm{Pd}$ profile of drug is required to combact the development of resistance. E.meningoseptica and S.maltophilia are the emerging NFGN pathogen due to misuse of antibiotics. cotrimaxole can be used effectively for treatment of S.maltophilia infections and MIC based treatment for ICU based resistant E.meningoseptica infections.

\section{References}

1. Chawla R. Epidemiology, etiology, and diagnosis of hospital-acquired pneumonia and ventilator-associated pneumonia in Asian countries. Am J Infect Cont. 2008; 34:S93-9.

2. Barbier F, Andremont A, Wolff M, Bouadma L. Hospital-acquired pneumonia and ventilator-associated pneumonia: recent advances in epidemiology and 
management. Curr Opin Pulm Med. 2013;19:216-28.

3. Sopena N, Heras E, Casas I, Bechini J, Guasch I, Pedro-Botet ML, et al. Risk factors for hospital-acquired pneumonia outside the intensive care unit: a casecontrol study. Am J Infect Control. 2014; $42: 38-42$.

4. In: Winn W Jr, Allen S, Janda W, Koneman E, Procop G, Schreckenberger $\mathrm{P}$, et al., editors. Nonfermenting Gram negative bacilli. In: Koneman's color Atlas and textbook of Diagnostic Microbiology. $6^{\text {th }}$ ed. USA: Lippincott Williams and Wilkins Company; 2006. p. 305-91.

5. Munoz-Price LS, Weinstein RA. Acinetobacter infection. $N$ Engl $J$ Med 2008;358:1271-81.

6. Wayne, Pa: Clinical and Laboratory Standards Institute; 2011. Clinical and Laboratory Standards Institute. Performance Standards for Antimicrobial Susceptibility Testing;21th Informational Supplement (M100-S21

7. Goossens H. Susceptibility of multi-drugresistant Pseudomonas aeruginosa in intensive care units: results from the European MYSTIC study group. Clin Microbiol Infec. 2003;9:980-3.

8. Gales AC, Jones RN, Forward KR et al. Emerging importance of multidrug-resistant Acinetobacter species and Stenotrophomonas maltophilia as pathogens in ill patients: geographic patterns, features, and trends in the SENTRY Antimicrobial Surveillance Program (1997-1999). Clin Infect Dis 2001; 32 Suppl 2:S104-13

9. Ridhima wadhwa1, yash sharma1, renuka pandey upadhyay2, kumud bala1* nosocomial infection by non-fermenting gram negative bacilli in tertiary care hospital: screening and cure international journal of pharmacy and pharmaceutical sciences issn- 0975-1491 vol 8, iss3, 2016
10. Sarika kombade1,Gopal N. Agrawal2 study of multidrug resistant nonfermenting gram-negative bacilli in intensive care unit, nagpur .Indian $j$ microbiol $R$ es2015;2(2):120-125

11. Kalidas Rit, Falguni Nag, Hirak Jyoti Raj†, PK MaityłPrevalence and Susceptibility Profiles of Nonfermentative Gram-negative Bacilli Infection ina Tertiary Care Hospital of Eastern India. Indian Journal of Clinical Practice, Vol. 24, No. 5, October 2013

12. Deepak Juyal, Rajat Prakash, Shamanth A. Shanakarnarayan, Munesh Sharma, Vikrant Negi, Neelam SharmaPrevalence of non-fermenting gram negative bacilli and their in vitro susceptibility pattern in a tertiary care hospital of Uttarakhand: A study from foothills of Himalayas.Saudi Journal for Health Sciences - Vol 2, Issue 2, May-Aug 2013.

13. Aoife Howard, $\uparrow$ Michael O’ Donoghue, $\uparrow$ Audrey $P$. Feeney, and Roy D. SleatorAcinetobacter baumannii An emerging opportunistic pathogen. Virulence. 2012 May 1; 3(3): 243-2

14. Oliveira MS, Prado GV, Costa SF, Grinbaum RS, Levin AS. Ampicillin/ sulbactam compared with polymyxins for the treatment of infections caused by carbapenem-resistant Acinetobacter spp. J Antimicrob Chemother 2008;61:1369-75.

15. Gilardi GL. Antimicrobial susceptibility as a diagnostic aid in the identification of nonfermenting gram negative bacteria. Appl Microbiol 1971;22(5):821-3.

16. Li Zhang, Xian-Zhi Li, and Keith Poole* Multiple Antibiotic Resistance in Stenotrophomonas maltophilia: Involvement of a Multidrug Efflux System. Antimicrob Agents Chemother. 2000 Feb; 44(2): 287-293.

17. Jones RN. 2010. Microbial etiologies of hospital-acquired bacterial pneumonia and 
ventilator-associated bacterial pneumonia. Clin. Infect. Dis. 51:S81-S87

18. M. S. Ratnamani and Ratna Rao Elizabethkingia meningoseptica: Emerging nosocomial pathogen in bedside hemodialysis patientsIndian J Crit Care Med. 2013 SepOct; 17(5): 304-3078.

19. Jean SS1, Lee WS2, Chen FL2, Ou TY2, HsuehP R3. Elizabethkingia meningoseptica: an important emerging pathogen causing healthcare-associated infections. J Hosp Infect. 2014 Apr;86 (4):244-9.

20. Balm MN1, Salmon S, Jureen R, Teo C, Mahdi R, Seetoh T, Teo JT, Lin RT, Fisher DA.Bad design, bad practices, bad bugs: frustrations in controlling an outbreak of Elizabethkingiameningosepticain intensive care units.J Hosp Infect. 2013 Oct;85(2):134-40. 